\title{
Whole Planet Cooling and the Radiogenic Heat Source Contents of the Earth and Moon
}

\author{
Gerald Schubert and DaVid STEVEnson \\ Department of Earth and Space Sciences, University of California, Los Angeles, California 90024 \\ Patrick Cassen
}

NASA Ames Research Center, Moffett Field, California 94035

\begin{abstract}
It is widely believed that the surface heat flows of the earth and moon provide good measures of the total amounts of radioactives in these bodies. Simple thermal evolution models, based on subsolidus whole mantle convection, indicate that this may not be the case. These models have been constructed assuming an initially hot state, but with a wide variety of choices for the parameters characterizing the rheology and convective vigor. All models are constrained to be consistent with present-day surface heat fluxes, and many of the terrestrial models are consistent with the mantle viscosities indicated by postglacial rebound. For the earth the acceptable models give a radiogenic heat production that is only $65-$ $85 \%$ of the surface heat output, the difference being due to secular cooling of the earth (about $50^{\circ}-100^{\circ} \mathrm{C}$ per $10^{9}$ years in the upper mantle). It is argued that the actual heat generation may be substantially less, since the models omit core heat, upward migration of heat sources, possible layering of the mantle, and deviations from steady convection. Geochemical models which are near to chondritic (apart from potassium depletion) are marginally consistent with surface heat flow. In the lunar models, heat generation is typically only $70-80 \%$ of the surface heat flow, even with allowance for the strong near-surface enhancement of radioactives. Despite the simplicity of the models the persistence of a significant difference between heat generation and heat output for a wide range of parameter choices indicates that this differ ence is real and should be incorporated in geochemical modeling of the planets.
\end{abstract}

\section{INTRODUCTION}

After the discovery of radioactivity but before the widespread acceptance of mantle convection and plate tectonics, cooling of the earth was thought to contribute substantially to the geothermal heat flux. Holmes [1916] attributed one quarter of the heat output to original heat, the rest being radiogenic, and Slichter [1941] stressed the large thermal inertia of the earth and the possibility of a large difference between heat generation and heat output.

More recently, the acceptance of the idea that there are plate and mantle convective motions with time scales much less than the age of the earth has led to the view that there is essentially a steady state in which heat production and heat loss through the surface are almost balanced [Tozer, 1965; Turcotte and Oxburgh, 1972]. This assumption has been made in discussions of the thermal state of the moon [Runcorn, 1962] and in attempts to estimate the abundances of uranium, thorium, and potassium in the earth and moon [Langseth et al., 1976].

However, the 'steady state' assumption only implies that heat generated deep within the body can be transported to the surface in a time much smaller than the age of that body; it does not imply equality of heat generation and heat output. This can be demonstrated by the following contradiction. Suppose heat generation and heat output were always equal. Since heat generation decreases with time because of the decay of radiogenic heat sources, the heat output also decreases with time. Convection experiments indicate that there is a positive correlation between the heat flux out of a convecting fluid layer and the temperature drop driving the convection. It follows that a decreased heat output implies a decreased temperature within a convecting planet. By the first law of thermodynamics this decrease in the planetary heat

Copyright $(\mathcal{1} 1980$ by the American Geophysical Union. content must contribute to the heat flux. This is in addition to the instantaneous radiogenic heat generation, so the original assumption is necessarily violated. This argument makes no explicit assumptions concerning the form of convection, and the inequality of heat generation and heat output occurs regardless of the time scale associated with convective overturn.

Estimates of the magnitude of this inequality depend on how one models the heat transfer and on a number of parameters which characterize the primordial and present states of the planets. The calculations reported here assume subsolidus whole mantle convection and demonstrate that there is a significant difference between heat generation and heat output for a wide range of plausible parameter values. The calculations are conservative in the sense that they almost certainly underestimate this difference, at least for the case of the earth. Nevertheless, we find that typically $15-35 \%$ of the geothermal heat flux can be attributed to cooling of the earth and that a similar difference between heat output and heat generation may apply for the moon. This difference should be incorporated in any estimate of the radiogenic elemental abundances of these planets.

A number of recent investigations [Sharpe and Peltier, 1978; Schubert, 1979; Schubert et al., 1979a, b; Stevenson and Turner, 1979; Cassen et al., 1979] have already indicated the likelihood of a significant difference, but in each instance the models chosen were from a narrow range of all conceivable models. Our purpose here is not to advocate a particular numerical value for the difference but to show that a significant difference is almost unavoidable. Davies [1980] and F. D. Stacey (unpublished manuscript, 1979) have independently reached the same conclusion.

\section{THE MODELS}

We shall first describe our models for the earth and then indicate how they are modified for the moon. We consider a 
simple spherical shell model of the mantle which has a viscosity $\nu$ and absolute temperature $T$ related according to

$$
\nu=\bar{\nu} \exp (A / T)
$$

where $\bar{\nu}$ and $A$ are constants. The temperature should be thought of as an 'average' mantle temperature; the significance of this average will be discussed later. Decaying radiogenic heat sources are assumed to produce energy at the rate $Q$ per unit volume, where

$$
Q=Q_{0} e^{-\lambda t}
$$

and $Q_{0}$ and $\lambda$ are constants. The lower boundary of the mantle has a radius $R_{c}$ and is assumed to be insulating. This is a simplifying assumption which neglects the heat that must necessarily be escaping from the core. This may be only about $10 \%$ of the surface heat loss [Stacey, 1977a], but if any part of it is due to core cooling (or inner core solidification), then this can only increase our estimate of the difference between heat generation and heat production.

A heat balance for the mantle gives

$$
\rho c\left(R_{m}{ }^{3}-R_{c}{ }^{3}\right) \dot{T}=-3 R_{m}{ }^{2} q+Q\left(R_{m}{ }^{3}-R_{c}{ }^{3}\right)
$$

where $\rho$ is the density, $c$ is the specific heat, $R_{m}$ is the outer radius of the mantle shell, $q$ is the heat flux from the mantle (which is less than the surface heat flow, primarily because of the radioactivity of continental crust), and $T$ is the time rate of change of $T$.

It is assumed that the heat flow from a vigorously convecting mantle exceeds the conductive heat flux by a multiplicative factor that is proportional to a fractional power of the Rayleigh number $R a$. The Rayleigh number is defined here as

$$
R a \equiv \frac{g \alpha\left(T-T_{s}\right)\left(R_{m}-R_{c}\right)^{3}}{\kappa \nu}
$$

where $T_{s}$ is the surface temperature, $\alpha$ is the coefficient of thermal expansion, $g$ is the acceleration of gravity, and $\kappa$ is the thermal diffusivity.

This definition of $R a$ is equivalent to that used in numerous studies of a convecting plane layer heated from below (see the references summarized by Schubert et al. [1979b]). In these instances the relationship between $q$ and $R a$ is of the form [Schubert, 1979; Schubert et al., 1979b]

$$
q=\frac{k\left(T-T_{s}\right)}{\left(R_{m}-R_{c}\right)}\left(\frac{R a}{R a_{c r}}\right)^{\beta}
$$

where $k$ is the thermal conductivity, $R a_{c r}$ is the critical value of $R a$ for the onset of convection, and $\beta$ is a dimensionless constant, typically around 0.3 in laboratory experiments. A numerical constant of proportionality often included in relationships of the form of (5) has been incorporated into the critical Rayleigh number.

The present applications differ from the plane layer heated from below in four respects. First, we consider an insulating lower boundary, so the temperature drop entering the definition of $R a$ is the temperature difference between the convecting region and the surface. Second, we consider a fluid heated from within. The laboratory experiments on internally heated fluid layers summarized by Schubert et al. [1979b], and the numerical experiments of McKenzie et al. [1974] show that the heat flow can still be parameterized in the form given by (5), using a Rayleigh number based on the actual temperature drop between the convecting region and the surface. It is al- ternatively possible to define a Rayleigh number based on the heat generation rate (the choice made by Turcotte et al. [1979]). These differences of definition can be encompassed by choosing a range of possible values of $R a_{c r}$ and $\beta$, as we do later. Third, we consider a spherical geometry. The numerical experiments on convection in spheres and spherical shells summarized by Schubert et al. [1979b] show that the relationship between $q$ and $R a$ (equation (5)) still applies with $\beta \approx 0.3$. The appropriate value of $R a_{c r}$ is somewhat dependent on geometry, however. Fourth, we have not allowed for the significantly nonzero adiabatic temperature gradient. However, the correction (which involves subtracting the appropriately averaged effect of the gradient from $T$ in the Rayleigh number) is unimportant in comparison with the uncertainties in the physical parameters.

The parameterization chosen here has been discussed in detail by Schubert et al. [1979b]. They showed that (4) and (5) give the correct form of the dependence of convective heat flow on Rayleigh number in both the limits of no heat generation (i.e., cooling from above) and steady internal heating. They also showed that the method describes the essential behavior of time-dependent systems in which the internal heating is suddenly changed. Experiments on fluids with a highly temperature-dependent viscosity [Booker, 1976] and theoretical calculations for a non-Newtonian rheology [Parmentier et al., 1976] indicate that the above parameterization may even be appropriate for the very complicated rheology of the mantle. However, the formulae are not exact; their accuracy must be evaluated by careful comparisons with appropriate experiments and numerical results [Schubert et al., 1979b]. For instance, if the value of $R a_{c r}$ is chosen so that (5) agrees exactly with Rossby's [1969] experiments on Bénard convection in water, the heat flow from a layer of internally heated water would be somewhat underestimated by the parameterization method, according to the experiments of Kulacki and Emara [1977]. For this reason, together with the uncertainties associated with geometry, boundary conditions, and other factors that distinguish planetary mantles from the laboratory and numerical experiments available for study, a range of parameter values must be explored. We will show that our conclusion-that a substantial fraction of the heat emanating from the earth and moon is due to the cooling of these bodies - is insensitive to the parameter values chosen.

Equations (1)-(5) provide a first-order differential equation for the temperature as a function of time $t$. Only $T(0)$, an initial condition on the mantle temperature, is required to find $T(t)$. Calculations were carried out for a broad range of the parameters $Q_{0}, \bar{\nu}, A, \beta, R a_{c n}$ and $T(0)$. For the remaining parameters we chose $\lambda=1.42 \times 10^{-17} \mathrm{~s}^{-1}, \kappa=10^{-2} \mathrm{~cm}^{2} \mathrm{~s}^{-1}, k=$ $10^{-2} \mathrm{cal} \mathrm{cm}^{-1} \mathrm{~s}^{-1} \mathrm{~K}^{-1}, \rho c=1 \mathrm{cal} \mathrm{cm}^{-3} \mathrm{~K}^{-1}, \alpha=3 \times 10^{-5} \mathrm{~K}^{-1}$, $g=10^{3} \mathrm{~cm} \mathrm{~s}^{-2}, T_{s}=273 \mathrm{~K}, R_{c}=3.471 \times 10^{8} \mathrm{~cm}$, and $R_{m}=$ $6.271 \times 10^{8} \mathrm{~cm}$. The value of $\lambda$ corresponds to the time-averaged decay constant for a chondritic composition and may be too large if the earth's mantle is depleted in potassium. Some models were constructed with different values of $\lambda$ or with (2) replaced by an appropriate mixture of ${ }^{40} \mathrm{~K},{ }^{235} \mathrm{U},{ }^{238} \mathrm{U}$, and ${ }^{232} \mathrm{Th}$; although the numerical results were changed, the final conclusions were not affected. The choice of the earth's surface temperature for $T_{s}$ the coldest temperature in the convecting system, acknowledges the important role' of sea floor spreading and subduction in transporting heat out of the earth.

The parameters $R a_{c r}$ and $\beta$ characterize the convective effi- 
ciency and are nominally 1100 and 0.3 , respectively. However, the boundary conditions and dynamic regime of the real mantle may not correspond closely to any available experimental or theoretical results, and in recognition of this we have considered models in which $R a_{c r}$ is as low as $10^{2}$ or as high as $10^{6}$ and $\beta$ is as low as 0.175 or as high as 0.5 . The values of $\bar{\nu}$ and $A$ entering the rheological law are poorly known, but it is generally agreed that the rheology is very strongly temperature dependent, with $A / T \sim 20$ to within a factor of 2 [Oxburgh and Turcotte, 1978]. The values of $A$ and $\bar{\nu}$ are constrained by the requirements that the present-day viscosity is around $10^{21}-10^{23} \mathrm{~cm}^{2} \mathrm{~s}^{-1}$ [Cathles, 1975; Peltier, 1976] and the present-day average mantle temperature is around 2000-3000 K [Stacey, 1977b]. Many (but not all) of the models constructed satisfied these constraints. In each model, $Q_{0}$ is adjusted to ensure that the heat flow from the mantle is about

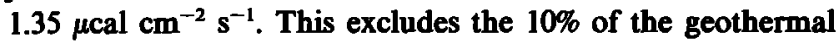
heat flux that can be attributed to radioactive elements in the continental crust [Oxburgh and Turcotte, 1978; Pollack and Chapman, 1977].

The remaining parameter $T(0)$, the initial mantle temperature, is poorly known but has little effect on the final results. This assertion is quantified in the next section, but it can be understood as follows. Suppose, first, that the planet starts out much hotter than its present temperature. This is likely for the earth, where early and rapid core formation may have produced a mantle temperature which was greater than the present value by $50-100 \%$ [Shaw, 1978]. In these circumstances, mantle convection is initially very vigorous, primarily because of the strong temperature dependence of the viscosity. In this early transieni phase, most of the excess heat content is eliminated. Subsequently, the ratio of heat output to heat generation remains roughly constant.

Suppose, instead, that the planet starts out cold. Since conduction is inefficient, the planet heats up internally, and the heat output is initially much less than the heat generation. For realistic choices of the radiogenic heat sources, convection is initiated in less than a billion years, and the temperature reaches a maximum in about 2 b.y. (at which time, heat output equals heat generation). Subsequently, the decay of the radiogenic heat sources ensures that the temperature decreases and the heat output exceeds heat generation.

The unimportance of $T(0)$ is thus a consequence of three factors: sufficient radiogenic heating to provide a hot planet in much less than $4.5 \times 10^{9}$ years, even if the planet is initially cold; a strongly temperature-dependent viscosity (which ensures rapid elimination of excess heat content); and vigorous present-day convection (which ensures that memory of the initial state is lost). The present difference between heat generation and heat output is obtained from previously generated heat. In the absence of detailed information concerning the early evolution the excess heat output cannot be labeled as 'primordial,' since it could also be the consequence of radiogenic heating in a previous epoch. The results reported here for the earth are for $T(0)=3273 \mathrm{~K}$, since we consider that a hot start to the evolution is most likely. However, a comparison is also made with a cold model in which $T(0)=300 \mathrm{~K}$.

The single temperature $T$ in the models is primarily determined by the rheological law, in accordance with the arguments of Tozer [1965]. This means that for each choice of $A$ and $\bar{\nu}, T$ will adjust so as to maintain the mantle viscosity required for transferring the specified heat flux. Since the cause for the difference between heat output and heat generation is the large heat content of the entire earth, it is also essential that $T$ be an average temperature in the following sense:

$$
T=\frac{\int \rho c T(\mathbf{r}) d V}{\int \rho c d V}
$$

where $T(r)$ is the actual mantle temperature at position $\mathbf{r}$ and the integrals extend over the mantle volume. Stacey's [1977b] thermal model of the earth gives $T \simeq 2550 \mathrm{~K}$ for the present day, but there are large uncertainties in the thermal structure of the deep mantle both as inferred from observation and from thermal history calculations. $O$. L. Anderson (unpublished manuscript, 1979) finds that the average mantle temperature is in the range $2200-2500 \mathrm{~K}$.

The primary difference between convection in the earth and the moon is that the lunar lithosphere does not participate in the convection. It is also likely that the near-surface concentration of radiogenic heat sources is greater than on earth. These differences make the lunar models more complicated, although the final conclusions are similar to those for the earth.

Equations (3)-(5) still apply to the moon, except that $T_{s}$ is replaced by $T_{b}$ the constant temperature at the base of the lithosphere, and the core radius $R_{c}$ is set equal to zero. The presence of a small iron core would not significantly change the results. The lithosphere thickens with time as the heat flux decreases, and this is incorporated by allowing relatively hot mantle material to 'freeze' at the lithospheric base. The lithosphere thickness $l$ then satisfies

$$
\rho c\left(T-T_{l}\right) d l / d t=q_{l}-q
$$

where $q$ is the heat flow from the mantle into the lithosphere and $q_{l}$ is the heat flow immediately above the lithospheremantle boundary.

The distribution of heat sources with depth is chosen to be

$$
\begin{aligned}
& Q=Q_{0} e^{-\lambda t}(1-\delta)\left[1+B \exp \left(-\frac{\gamma[R-r]}{R}\right)\right] \quad R-l<r<R \\
& Q=Q_{0} e^{-\lambda r}(1-\delta) \quad 0<r<R-l
\end{aligned}
$$

where $R$ is the radius of the moon, $B$ is chosen to fit the observed surface enhancement of radioactive elements [Metzger et al., 1974], $\gamma$ is chosen to be consistent with the crustal thickness, and $\delta$ is chosen so that the whole moon average of $Q$ is $Q_{0} e^{-\lambda t}$. The parameter $\delta$ is therefore a measure of the average depletion of the lunar interior. Since $l$ changes with time, $\delta$ also changes with time, so this model crudely represents the early upward migration of heat sources. Once $\gamma l / R \gg 1$, however, the discontinuity in $Q$ at $r=R-l$ becomes negligible, and so does the upward migration. In the models described here, $B=14$ (corresponding to a surface enhancement of about 6.1), $\gamma=29$ (corresponding to an $e$-folding depth of 60 $\mathrm{km}$, comparable to the crustal thickness), and $\delta \simeq 0.57$ at the present day (so that almost $60 \%$ of the heat generation is in the crust). In the models, $\delta$ starts at zero because $l(t=0)$ is chosen to be very small, but it approaches its present value after only $5 \times 10^{8}$ to $1 \times 10^{9}$ years.

The temperature profile in the lithosphere is approximated by a conductive steady state. This is an adequate approximation so long as the time scale for thermal diffusion through the lithosphere $\left(\sim l^{2} / K\right)$ is less than the time scale associated with lithosphere thickening $(-l(d t / d l))$ or the time scale of radio- 


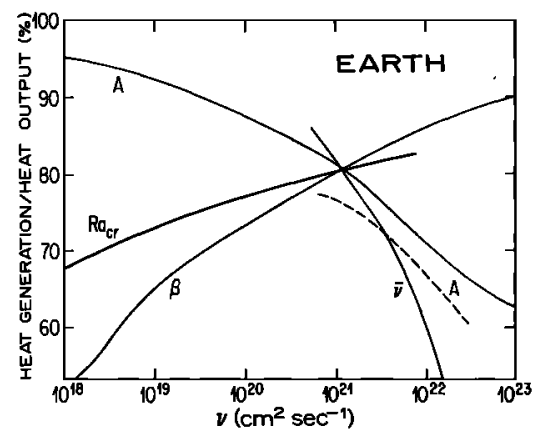

Fig. 1. Summary of earth models showing the ratio of heat generation to heat output versus present-day mantle viscosity $\nu$ for a variety of parameter choices. The point common to all the solid curves is the nominal case for which $A=5.6 \times 10^{4} \mathrm{~K}, R a_{c r}=1100, \bar{\nu}=1.65 \times 10^{6}$ $\mathrm{cm}^{2} \mathrm{~s}^{-1}$, and $\beta=0.3$. Each of the solid curves is labeled by the one parameter whose value is varied from the nominal case. Proceeding from left to right along each curve, the respective parameter variations are $2 \times 10^{4} \mathrm{~K} \leq A \leq 1.5 \times 10^{5} \mathrm{~K}, 10^{6} \geq R a_{c r} \geq 10^{2}, 0.15 \leq \beta \leq$ 0.5 , and $16.5 \mathrm{~cm}^{2} \mathrm{~s}^{-1} \leqslant \bar{\nu} \leqslant 1.65 \times 10^{13} \mathrm{~cm}^{2} \mathrm{~s}^{-1}$. The dashed line is for $\bar{\nu}=1.65 \times 10^{9} \mathrm{~cm}^{2} \mathrm{~s}^{-1}, \beta=0.3, R a_{c r}=1100$, and $4 \times 10^{4} \mathrm{~K} \leqslant A \leqslant 10^{5}$ $\mathbf{K}$.

active decay. These conditions begin to be violated in some models at about the present day $(l \sim 250 \mathrm{~km})$, but the resulting corrections to the models are not likely to modify our conclusions substantially. The steady state temperature profile is the solution of $-k \nabla^{2} T(r)=Q$ and has the form

$$
\begin{aligned}
T(r)=\frac{a}{r}+b-\frac{Q_{0} e^{-\lambda t}(1-\delta)}{k} & {\left[\frac{r^{2}}{6}+B \exp \left[-\frac{\gamma(R-r)}{R}\right]\right.} \\
\cdot & {\left.\left[\left(\frac{R}{\gamma}\right)^{2}-\frac{2}{r}\left(\frac{R}{\gamma}\right)^{3}\right]\right] }
\end{aligned}
$$

where $a$ and $b$ are chosen so that $T_{s}=T(R), T_{t}=T(R-l)$.

The upward heat flux at the lithosphere-mantle interface $q_{1}=-[k(d T / d r)]_{R-l,}$ the surface heat flux $-[k(d T / d r)]_{R}$, and equations (2)-(5) and (7) provide a complete specification for the solution of a pair of coupled first-order differential equations for $T(t)$ and $l(t)$. The initial values were chosen to be $T(0)=1773 \mathrm{~K}$ and $l(0)=0.1 \mathrm{~km}$. The results are relatively insensitive to these choices. The temperature $T_{t}$ at the lithospheric base was set at a constant value of $1073 \mathrm{~K}$. The parameters $\lambda, K, k, \rho c, \alpha$, and $T_{s}$ were the same as for the earth, $g$ $=160 \mathrm{~cm} \mathrm{~s}^{-2}$, and $R=1.740 \times 10^{8} \mathrm{~cm}$. The value of $Q_{0}$ was adjusted in each model so as to produce a present-day surface heat flow of $0.4 \mu \mathrm{cal} \mathrm{cm} \mathrm{cm}^{-2} \mathrm{~s}^{-1}$, consistent with the estimate of Langseth et al. [1976].

There is no accurate constraint on the viscosity of the lunar interior, although Meissner and Voss [1979] conclude from a consideration of moonquakes that values exceeding $3 \times 10^{21}$ $\mathrm{cm}^{2} \mathrm{~s}^{-1}$ at $700-\mathrm{km}$ depth and less than $3 \times 10^{20} \mathrm{~cm}^{2} \mathrm{~s}^{-1}$ at $1150-\mathrm{km}$ depth are likely. The models described here have present-day viscosities in the range $10^{20}-10^{23} \mathrm{~cm}^{2} \mathrm{~s}^{-1}$.

\section{RESULTS}

For the earth we chose a nominal case and then examined parameter variations therefrom. The nominal case was $A=$ $5.6 \times 10^{4} \mathrm{~K}, \beta=0.3, R a_{c r}=1100$, and $\bar{\nu}=1.65 \times 10^{6} \mathrm{~cm}^{2} \mathrm{~s}^{-1}$. The value of $Q_{0}$ was then adjusted to give a present-day mantle heat flux of $1.35 \mu \mathrm{cal} \mathrm{cm} \mathrm{cm}^{-2} \mathrm{~s}^{-1}$. For this nominal case, $Q=6.1 \times 10^{-15} \mathrm{cal} \mathrm{cm}^{-3} \mathrm{~s}^{-1}$ after $4.5 \times 10^{9}$ years, only $80 \%$ of the value of $Q$ that would be inferred from surface heat flow and the assumption that there is equilibrium between heat loss and heat production. The present-day viscosity for the nominal case is $1.5 \times 10^{21} \mathrm{~cm}^{2} \mathrm{~s}^{-1}$. The same results (to within $0.1 \%$ ) were obtained for $T(0)=300 \mathrm{~K}$ and $T(0)=3273 \mathrm{~K}$. It must be stressed that this nominal case has no special significance; it merely serves as a reference point for comparison.

Figure 1 shows the results for the ratio of present-day heat generation to present-day heat output (total heat flow from the mantle per unit volume of the mantle) for a large number of models in which each of the four parameters $A, \beta, R a_{c r}$ and $\bar{\nu}$ is varied from its nominal value. Thus the curve labeled $A$ corresponds to a set of models in which $\operatorname{Ra}_{c r} \beta$, and $\bar{\nu}$ are fixed at their nominal values, the correct present-day heat flux is obtained, and $A$ is allowed to vary. Similarly, the other three curves correspond to variations in each of the other three parameters. All of these curves pass through the single point which corresponds to the nominal case. This parameter study indicates that the substantial difference between heat output and heat generation exists even for parameter values that differ greatly from the nominal state. In a few models, more than one of the nominal parameters was changed; for example, the dashed line in Figure 1 corresponds to the nominal values of $R a_{c r}$ and $\beta$, but $\bar{\nu}=1.65 \times 10^{9} \mathrm{~cm}^{2} \mathrm{~s}^{-1}$, and $A$ varies between $4 \times 10^{4} \mathrm{~K}$ and $10^{5} \mathrm{~K}$. These models are of interest, since they produce average present-day mantle temperatures similar to those independently derived by Stacey [1977b] and they give present-day viscosities consistent with postglacial rebound [Cathles, 1975; Peltier, 1976].

The earth model of Turcotte et al. [1979] for a free upper boundary is equivalent to a model in our parameterization, for the choices $A=6.3 \times 10^{4} \mathrm{~K}, \bar{\nu} \simeq 1 \times 10^{3} \mathrm{~cm}^{2} \mathrm{~s}^{-1}, \beta=$ 0.333 , and $R a_{c r} \simeq 60$. For this model the difference between heat generation and heat output is only about $10 \%$, similar to the result of Turcotte et al. [1979]. However, this model is unrealistic, since it gives a mantle viscosity that is about an order of magnitude greater than the highest value indicated by postglacial rebound [Cathles, 1975; Peltier, 1976]. The temperature in this model is about $1600 \mathrm{~K}$, which Turcotte et al. [1979] interpret as a 'near surface reference temperature'; but as discussed before, it should properly be interpreted as a mantleaveraged temperature, since the cooling of the entire mantle contributes to the surface heat flow. In that case the value $1600 \mathrm{~K}$ would clearly be too low.

Figures 2-4 show the thermal state of the mantle model for the case $R a_{c r}=1100, \beta=0.3, \bar{\nu}=1.65 \times 10^{6} \mathrm{~cm}^{2} \mathrm{~s}^{-1}, A=7 \times$ $10^{4} \mathrm{~K}$, and $T(0)=3273 \mathrm{~K}$. Models with nearly the same parameter values (e.g., the one for $R a_{c r}=1100, \beta=0.3, \bar{\nu}=1.65$ $\times 10^{9} \mathrm{~cm}^{2} \mathrm{~s}^{-1}$, and $A=5.6 \times 10^{4} \mathrm{~K}$ ) yield very similar results. Figure 2 shows that the viscosity increases monotonically from $3.2 \times 10^{15} \mathrm{~cm}^{2} \mathrm{~s}^{-1}$ at the beginning of the thermal history

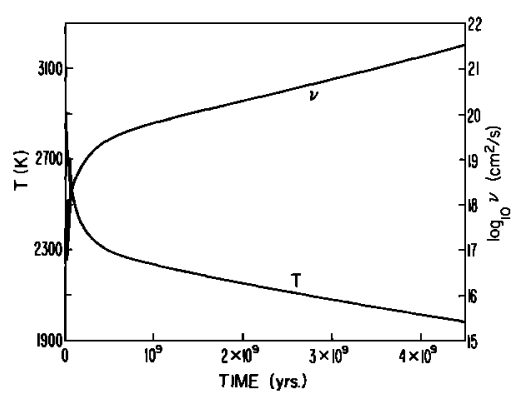

Fig. 2. Mantle viscosity $\nu$ and temperature $T$ versus time for the earth model in which $A=7 \times 10^{4} \mathrm{~K}, \bar{\nu}=1.65 \times 10^{6} \mathrm{~cm}^{2} \mathrm{~s}^{-1}, R a_{c r}=$ 1100 , and $\beta=0.3$. 


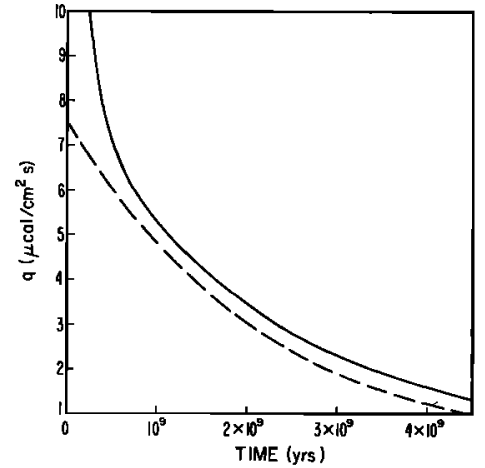

Fig. 3. Surface heat flow $q$ (solid line) and rate of internal heat generation per unit surface area (dashed line) versus time for the same earth model as in Figure 2; $q$ is $1.33 \mu \mathrm{cal} \mathrm{cm} \mathrm{cm}^{-2} \mathrm{~s}^{-1}$ at $t=4.5 \times 10^{9}$ years, and the heat generation is $75 \%$ of this.

to $3.4 \times 10^{21} \mathrm{~cm}^{2} \mathrm{~s}^{-1}$ after $4.5 \times 10^{9}$ years. The change in temperature is less than $50 \%$ because of the very strong temperature dependence of the viscosity. The solid line in Figure 3 shows the surface heat flow as a function of time. At $t=4.5 \times$ $10^{9}$ years it is $1.33 \mu \mathrm{cal} \mathrm{cm}^{-2} \mathrm{~s}^{-1}$. The dashed line is the heat flux that would occur if there were equilibrium between heat generation and heat output. Figure 4 shows how the Rayleigh number and the Nusselt number (the ratio of the heat flow from the convecting mantle to the conductive heat flow $k(T-$ $\left.\left.T_{s}\right) /\left(R_{m}-R_{c}\right)\right)$ of the vigorously convecting mantle decay with time. At present, the model has a Rayleigh number about $3 \times 10^{4}$ times critical, and about 20 times as much heat is carried through the mantle by convection as would be carried by conduction alone.

Models with a cold start $(T(0)=300 \mathrm{~K})$ have a very different evolution for the first 2 b.y. but are essentially indistinguishable from hot evolutions after 2.5 or 3 b.y. For the nominal case the ratio of heat generation to heat output is 146\% (cold start), 94\% (hot start) after 1 b.y.; 97\% (cold), 91\% (hot) after 2 b.y., and $86 \%$ (both) after 3 b.y. Models with a cold start are not described in detail, since they would require a very heterogeneous accretion, incompatible with our assumption of whole mantle convection.

A similar parameter study has been carried out for the lunar models, and the results are summarized in Figure 5. Here, the heat generation includes the crustal contribution, and the heat output is the total heat flow through the lunar surface. This contrasts with the heat generation'/heat output ratios for the models of Figure 1 which did not incorporate the relatively small crustal radioactivity. The nominal parameters

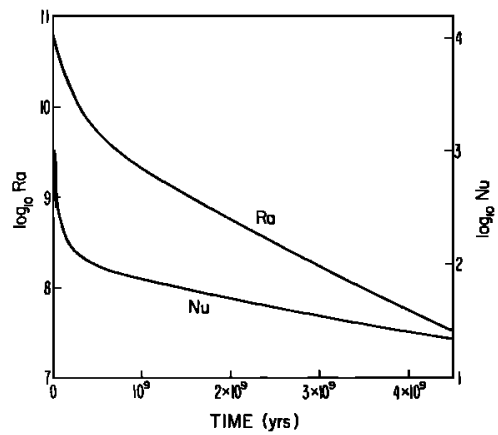

Fig. 4. Evolution of the Nusselt number $N u$ (ratio of total to conductive heat flux) and Rayleigh number $R a$ for the same earth model as in Figure 2. were the same as for the earth, except that $\bar{\nu}=4.5 \times 10^{6} \mathrm{~cm}^{2}$ $\mathrm{s}^{-1}$. As in Figure 1, each curve represents the variation of one of the four parameters $A, R a_{c n} \beta$, and $B$. Not all of the lunar models have been included in this figure. It was found, for example, that models in which $A=4 \times 10^{4} \mathrm{~K}$ and $\bar{\nu}$ was variable describe a curve that is almost coincident with the curve drawn for $\bar{\nu}$ fixed and $A$ variable. The models have also been restricted to those for which convection occurs throughout the evolution. Models for $A \geq 7 \times 10^{4} \mathrm{~K}$ were usually nonconvecting in the early evolution for the choice $T(0)=1773$ $K$. Since the moon is smaller than the earth, the effect of pressure on rheology is less important in the moon, and the appropriate value of $\boldsymbol{A}$ may be smaller, even if the lunar mantle has the same activation energy and activation volume as the earth's mantle.

In Figures 6-9, properties of the nominal lunar model $\left(R a_{c r}=1100, \beta=0.3, \nu=4.5 \times 10^{6} \mathrm{~cm}^{2} \mathrm{~s}^{-1}, A=5.6 \times 10^{4} \mathrm{~K}\right)$ are shown versus time. Figure 6 shows that this particular model starts out slightly cold and undergoes a very small temperature increase before cooling by about $150^{\circ} \mathrm{C}$ over the age of the moon. (This initial slight heating has no special significance; some models are completely monotonic.) The viscosity increases throughout almost all of the evolution and reaches a present-day value of $5.9 \times 10^{21} \mathrm{~cm}^{2} \mathrm{~s}^{-1}$, roughly consistent with the estimate of Meissner and Voss [1979].

Figure 7 shows the surface heat flux as a function of time (solid curve), while the dashed curve is the flux for equilibrium between heat generation and heat output. In the early evolution the heat flux is derived almost entirely from the thickening of the lithosphere and decreases in a way that is similar to the observed decrease of oceanic heat flow with age of the ocean floor on earth. Subsequently, the small but significant difference between heat output and heat generation is provided partly by thickening of the lithosphere and partly by decreasing the heat content of the deep interior. At the present day this model provides $q=0.406 \mu \mathrm{cal} \mathrm{cm}^{-2} \mathrm{~s}^{-1}$ and only requires $73 \%$ of the radiogenic heating that would be needed if there were equality of heat generation and heat output.

The variations of Rayleigh number and Nusselt number throughout the evolution are shown in Figure 8. In this model, $R a$ is about 100 times the critical value at the present day, and the convection is transporting only about 5 times more heat from the deep interior than conduction would. Figure 9 shows the evolution of the lithospheric thickness $l$ and

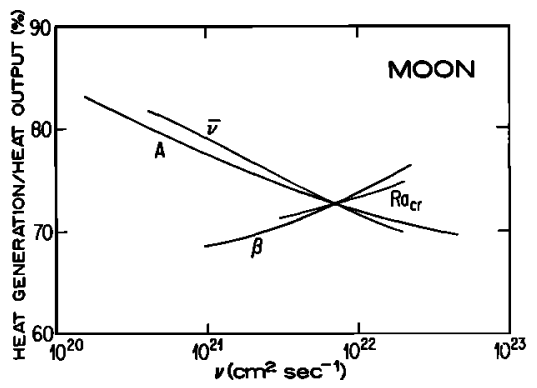

Fig. 5. Summary of moon models showing the ratio of heat generation to heat output versus present-day mantle viscosity $\nu$ for a variety of parameter choices. The point common to all the solid curves is the nominal case for which $A=5.6 \times 10^{4} \mathrm{~K}, \bar{\nu}=4.5 \times 10^{6} \mathrm{~cm}^{2} \mathrm{~s}^{-1}, R a_{c r}=$ 1100 , and $\beta=0.3$. Each curve is labeled by the one parameter which is varied from its nominal value. Proceeding from left to right along each curve, the respective parameter variations are $4 \times 10^{4} \mathrm{~K} \leqslant A \leqslant 7$ $\times 10^{4} \mathrm{~K}, 5 \times 10^{3} \geqslant R a_{c r} \geqslant 200,0.2 \leqslant \beta \leqslant 0.4$, and $4.5 \times 10^{2} \mathrm{~cm}^{2} \mathrm{~s}^{-1} \leqslant$ $\bar{i} \leqslant 4.5 \times 10^{8} \mathrm{~cm}^{2} \mathrm{~s}^{-1}$. 


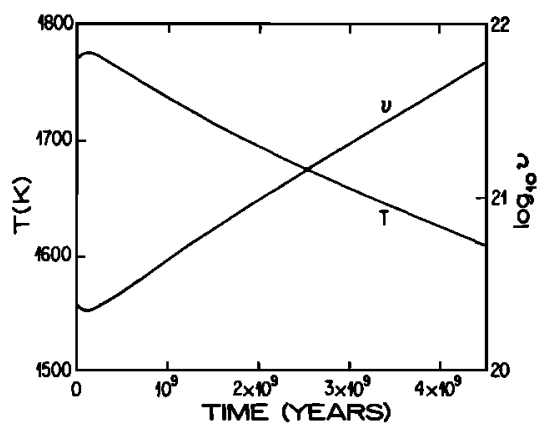

Fig. 6. Mantle viscosity $\nu$ and temperature $T$ versus time for the nominal moon model.

mantle depletion of radioactive elements $\delta$. In this crude model the lithosphere exceeds the present crustal thickness after the first few hundred million years, and all but $10 \%$ of the upward migration of radioactive elements has been completed in $5 \times 10^{8}$ years. At present, the lithospheric thickness is 290 $\mathrm{km}$ and the mantle has only $43 \%$ of the radioactive heat sources. In other models the lithospheric thickness was as little as $150 \mathrm{~km}$ and as great as $350 \mathrm{~km}$.

\section{DISCUSSION}

The heat content of the earth is immense: about $1 \times 10^{38}$ ergs, equal to about a 10-b.y. supply of the present geothermal heat flux. Only a very small fraction of this energy need escape to cause a significant difference between heat generation and heat output. The rate of escape is severely limited, primarily by the very strong temperature dependence of the viscosity, but the models described here indicate that the earth's heat content is decreasing by $2-4 \%$ per billion years, and this is enough to cause a significant difference. The inferred decrease in upper mantle temperature of between $50^{\circ}$ and $100^{\circ} \mathrm{C}$ per billion years is consistent with the presence of Archean komatiites whose source regions appear to have been at least $200^{\circ} \mathrm{C}$ hotter than the hottest source regions of present-day lavas [Green, 1972]. Quantitative explanation of these ultrabasic magmas may, however, require consideration of the evolving geochemical and tectonic environment [Weaver and Tarney, 1979] as well as our simple consideration of changing mantle temperature. Although the moon is a smaller body and has a smaller heat content relative to heat output, the difierence between heat generation and heat output is again large, perhaps because the moon has progressed further in its evolution and has a much thicker cold outer layer. (Figure 7 in-

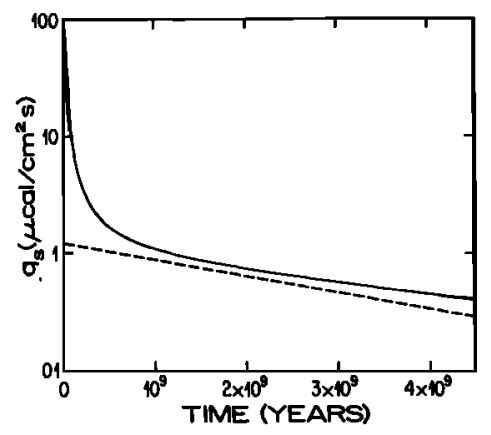

Fig. 7. Surface heat flow $q_{s}$ (solid line) and the rate of internal heat generation per unit surface area (dashed line) versus time for the nominal moon model; $q_{s}$ is $0.406 \mu \mathrm{cal} \mathrm{cm}^{-2} \mathrm{~s}^{-1}$ at $t=4.5 \times 10^{9}$ years, and the heat generation is $73 \%$ of this. dicates that the fractional difference between heat output and heat generation is a minimum after about 2 b.y. but is now steadily increasing.)

In contrast, McKenzie and Weiss [1975] concluded that the difference between heat generation and heat output for the earth is small. We agree with their conclusions that convection can efficiently eliminate excess heat (such as that which may have been present after core formation) and that the strong temperature dependence of the rheology prevents large changes in mantle temperature over geologic time. However, McKenzie and Weiss [1975] never calculated the difference between heat generation and heat output for whole mantle convection in the presence of a secularly decreasing heat source. In the only calculations similar to ours they considered a layer depth of $700 \mathrm{~km}$ and a temperature which characterizes the upper mantle rather than the 'average' mantle as discussed above. Together, these led them to a difference that was a factor of about 4 less than our estimates. It is not therefore inconsistent that they found less than $5 \%$ difference between heat generation and heat output [see McKenzie and Weiss, 1975, p. 146].

Although the models are very simplified descriptions of complex, convecting systems, it is difficult to conceive of plausible modifications to the models which would decrease the difference between heat output and heat generation. In the case of the earth especially we may have substantially underestimated the difference.

First, we have omitted the heat flux from the core. If the core is near adiabatic and has a short convective overturn time (as is required for a geodynamo), then it will respond essentially instantaneously to secular mantle cooling. This core cooling may be enough to drive the dynamo [Schubert et al., 1979a]. Freezing of the inner core may further enhance the nonradiogenic heat flow out of the core, because of latent heat and gravitational differentiation [Gubbins, 1976]. The thermal conductivity and adiabatic gradient of the core are poorly known, but a core to mantle heat flow of $10 \%$ of the total heat flow is possible [Schubert et al., 1979a], and this should be added to the previously calculated 15-35\% difference between heat generation and heat output.

Second, we have omitted the ongoing upward migration of radioactive elements. If the mantle is undergoing gradual depletion of heat sources, then the heat flux from the deep mantle is decreasing with time because of both migration and radioactive decay. This effect might add as much as $10 \%$ to the difference, but it is difficult to calculate without knowledge of the source depth(s) of basalts.

Third, we have assumed that the whole mantle is overturning. If the mantle is layered, it may still convect almost

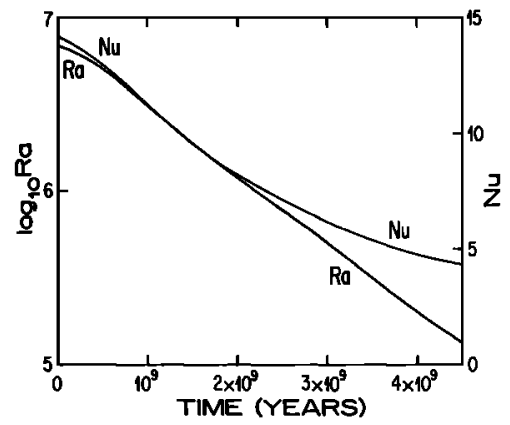

Fig. 8. Evolution of the Nusselt number $N u$ and Rayleigh number $R a$ for the nominal moon model. 


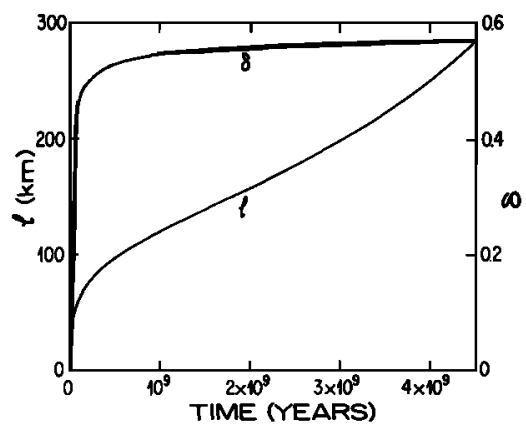

Fig. 9. Evolution of the lithospheric thickness $l$ and mantle radiogenic heat source depletion $\delta$ for the nominal moon model.

everywhere, but with little transport of material between layers [Stevenson and Turner, 1979; Richter and Johnson, 1974]. Under these circumstances, there is a thermal boundary layer between each of the layers, and the total heat content of the earth may be even higher than we assumed. This can only increase the difference between heat generation and heat output. A similar situation may arise in the moon, where geochemical and seismic models suggest a depleted, low-density upper mantle to depth $\mathbf{4 0 0}-\mathbf{4 8 0} \mathrm{km}$ overlying a denser, primordial lower mantle [Goins et al., 1979].

Fourth, we have neglected the finite convective overturn time in the mantle by assuming that the present-day radiogenic heat production is instantaneously available for the surface heat output. Daly and Richter [1978] have suggested that there could be a large deviation from this quasi steady state even though the convective overturn time $\left(\sim 10^{8}\right.$ years at present for the earth) is much less than the radioactive decay time. Their model assumes a time-independent cell structure for the convection, which may not be applicable to the present earth, but any time lag between heat generation and heat output can only increase the difference. This effect could be even larger in the moon, where the convective overturn time may now be approaching a billion years.

The models described in this paper, together with the above arguments, strongly suggest that at least $25 \%$, and perhaps even as much as $50 \%$, of the geothermal heat output is not attributable to present-day radiogenic heat production. A number of geochemists (see, for example, Ringwood [1975], Taylor [1979], and $O^{\prime} N i o n s$ et al. [1979]) have reached similar conclusions from purely geochemical arguments. A potassium-depleted but otherwise chondritic earth (with $K / \mathrm{U}=10^{4}$ ) would produce about $50 \%$ of the present geothermal heat flux at most. If the mantle is layered, with part of the mantle enriched in heat sources but not contributing to the formation of surface rocks, then the current geochemical estimates may be too low. Nevertheless, the models and arguments presented here indicate that there is not necessarily any discrepancy between current geochemical and geophysical modeling of the earth or moon. Further work in both disciplines should establish more precise limits on the difference between heat generation and heat output.

Acknowledgments. This research was supported in part by NSF grant EAR77-15198 and by the Planetology Geology Program, Office of Space Science, NASA grant NGR05-007-317. We thank Ross Taylor and Don Turcotte for helpful discussions.

\section{REFERENCES}

Booker, J. R., Thermal convection with strongly temperature-dependent viscosity, J. Fluid Mech., 76, 741-754, 1976.
Cassen, P., R. T. Reynolds, F. Graziani, A. Summers, J. McNellis, and L. Blalock, Convection and lunar thermal history, Phys. Earth Planet, Interiors, 19, 183-196, 1979.

Cathles, L. M., III, The Viscosity of the Earth's Mantle, Princeton University Press, Princeton, N. J., 1975.

Daly, S. F., and F. M. Richter, Convection with decaying heat sources: A simple thermal evolution model (abstract), in Lunar and Planetary Science IX, pp. 213-214, Lunar and Planetary Science Institute, Houston, Tex., 1978.

Davies, G. F., Thermal histories of convective earth models and constraints on radiogenic heat production in the earth, J. Geophys. Res., 85 , in press, 1980.

Goins, N. R., M. N. Toksöz, and A. M. Dainty, The lunar interior: A summary report (abstract), in Lunar and Planetary Science $X$, pp. 437-439, Lunar and Planetary Science Institute, Houston, Tex., 1979.

Green, D. H., Archean greenstone may include terrestrial equivalents of lunar maria?, Earth Planet. Sci. Lett., 15, 263-270, 1972.

Gubbins, D., Observational constraints on the generation process of the earth's magnetic field, Geophys. J. Roy. Astron. Soc., 47, 19-39, 1976.

Holmes, A., Radioactivity in the earth's thermal history, Geol. Mag., 3, 265-274, 1916.

Kulacki, F. A., and A. A. Emara, Steady and transient thermal convection in a fluid layer with uniform volumetric energy sources, $J$. Fluid Mech., 83, 375-395, 1977.

Langseth, M. G., S. J. Keihm, and K. Peters, Revised lunar heat flow values, Proc. Lunar Sci. Conf. 7th, 3143-3171, 1976.

McKenzie, D. P., and N. Weiss, Speculation on the thermal and tectonic history of the earth, Geophys. J. Roy. Astron. Soc., 42, 131174, 1975.

McKenzie, D. P., J. M. Roberts, and N. O. Weiss, Convection in the earth's mantle: Towards a numerical solution, J. Fluid Mech., 62, 465-538, 1974.

Meissner, R., and J. Voss, Viscosity limits and possible origin of tidal moonquakes (abstract), in Lunar and Planetary Science X, pp. 822824, Lunar and Planetary Science Institute, Houston, Tex., 1979.

Metzger, A. E., J. I. Trombka, R. C. Reedy, and J. R. Amold, Element concentrations from lunar orbital gamma-ray measurements, Proc. Lunar Sci. Conf. 5th, 1067-1078, 1974.

O'Nions, R. K., S. R. Carter, N. M. Evenson, and P. J. Hamilton, Upper mantle geochemistry, in The Sea, vol. VII, edited by E. Emiliani, Interscience, New York, in press, 1979.

Oxburgh, E. R., and D. L. Turcotte, Mechanisms of continental drift, Rep. Progr. Phys., 41, 1249-1312, 1978.

Parmentier, E. M., D. L. Turcotte, and K. E. Torrance, Studies of finite-amplitude non-Newtonian thermal convection, with application to convection in the earth's mantle, J. Geophys. Res., 81, 1839-1846, 1976.

Peltier, W. R., Glacial isostatic adjustment, II, The inverse problem, Geophys. J. Roy. Astron. Soc., 46, 669-705, 1976.

Pollack, H. N., and D. S. Chapman, Mantle heat flow, Earth Planet. Sci. Lett., 34, 174-184, 1977.

Richter, F. M., and C. E. Johnson, Stability of a chemically layered mantle, J. Geophys. Res., 79, 1635-1639, 1974.

Ringwood, A. E., Composition and Petrology of the Earth's Mantle, pp. 199-200, McGraw-Hill, New York, 1975.

Rossby, H. T., A study of Benard convection with and without rotation, J. Fluid Mech, 36, 309-335, 1969.

Runcom, S. K., Convection in the moon, Nature, 195, 1150-1151, 1962.

Schubert, G., Subsolidus convection in the mantles of terrestrial planets, Annu. Rev. Earth Planet. Sci., 7, 289-342, 1979.

Schubert, G., P. Cassen, and R. E. Young, Core cooling by subsolidus mantle convection, Phys. Earth Planet. Interiors, 20, 194-208, 1979a.

Schubert, G., P. Cassen, and R. E. Young, Subsolidus convective cooling histories of terrestrial planets, Icarus, 38, 192-211, $1979 b$.

Sharpe, H. N., and W. R. Peltier, Parameterized mantle convection and the earth's thermal history, Geophys. Res. Lett., 5, 737-740, 1978.

Shaw, G. H., Effects of core formation, Phys. Earth Planet. Interiors, 16, 361-369, 1978.

Slichter, L. B., Cooling of the earth, Geol. Soc. Amer. Bull., 52, 561$600,1941$.

Stacey, F. D., Physics of the Earth, p. 198, John Wiley, New York, $1977 a$.

Stacey, F. D., A thermal model of the earth, Phys. Earth Planet. Interiors, $15,341-348,1977 b$. 
Stevenson, D. J., and J. S. Turner, Fluid models of mantle convection, in The Earth, It's Origin, Evolution and Structure, edited by $\mathrm{M}$. McElhinny, pp. 227-263, Academic, New York, 1979.

Taylor, S. R., Relative refractory and volatile element contents of the earth and the moon (abstract), in Lunar and Planetary Science $X$, pp. 1217-1218, Lunar and Planetary Science Institute, Houston, Tex., 1979.

Tozer, D. C., Heat transfer and convection currents, Phil. Trans. Roy. Soc. London, 258, Ser. A, 252-271, 1965.

Turcotte, D. L., and E. R. Oxburgh, Mantle convection and the new global tectonics, Annu. Rev. Fluid Mech., 4, 33-68, 1972.
Turcotte, D. L., F. A. Cook, and R. J. Willeman, Parameterized convection within the moon and the terrestrial planets, Proc. Lunar Planet. Sci. Conf. 10th, 1979.

Weaver, B. L., and J. Tarney, Thermal aspects of komatiite generation and greenstone belt models, Nature, 279, 689-692, 1979.
(Received October 16, 1979;

accepted January 14, 1980.) 